\title{
Oral Rehabilitation in Irradiated Patients: Implant- or Tooth-Supported Fixed Prosthesis? A Clinical Report
}

\section{Somayeh Allahyari*}

Department of Dental Technology, School of Dentistry, Tehran University of Medical Sciences, Tehran, Iran

\begin{tabular}{l}
\hline Article Info \\
\hline Article type: \\
Case Report Article \\
\hline Article History: \\
Received: 22 September 2018 \\
Accepted: 23 December2018 \\
Published: 30 August 2019 \\
\hline * Corresponding author: \\
Department of Dental Technology, School of \\
Dentistry, Tehran University of Medical \\
Sciences, Tehran, Iran \\
Email: somayeh.al@gmail.com
\end{tabular}

A B S T R A C T

Cranial radiotherapy has several side effects. One of the most important complications is radiation caries that endangers the treatment prognosis. In the literature, the use of crowns and bridges for irradiated patients has been suggested as a contraindication. In addition, due to the risk of osteoradionecrosis (ORN), there are doubts about tooth extraction and implant placement. Here, we present a treatment sequence and recalls for an irradiated young patient. For irradiated patients, it is recommended to replace teeth with implants when there is no possibility for supragingival prosthetic margin.

Keywords: Cranial Irradiation; Osteoradionecrosis; Dental Implants; Dental Caries

Cite this article as: Allahyari S. Oral Rehabilitation in Irradiated Patients: Implant- or Tooth-Supported Fixed Prosthesis? A Clinical Report. Front Dent. 2019;16(4):319-324. doi:

\section{INTRODUCTION}

Cranial cancers are often treated using radiation therapy (RT), with some adverse effects during and after irradiation, such as xerostomia (90\%), mucositis $(60 \%)$, postirradiation dental decay (50\%), and osteoradionecrosis (ORN; 15\%) [1]. Although most of these complications are manageable, they can influence dental treatment choices and prognosis. Among the complications, radiation caries is more challenging for restorative dentists. Radiation caries is one of the early complications that occur after RT. Chemical and microbial changes in the mouth can begin during 3 months after radiotherapy, resulting in caries that may spread to all teeth during 3 years $[2,3]$. Xerostomia leads to change of $\mathrm{pH}$ and the viscosity of saliva as well as an increased rate of caries $[4,5]$.

RT also has a direct effect on tooth structure through weakening the enamel and dentin structure, compromising the use of bonding materials $[6,7]$. There is a contraindication for fixed prosthetic treatment in irradiated patients in the literature [1,2]. Even with good oral hygiene, margins of crowns and bridges are prone to caries; therefore, if a patient insists on fixed prosthetic treatments, it is

This work is published as an open access article distributed under the terms of the Creative Commons Attribution 4.0 License (http://creativecommons.org/licenses/by-nc/4). Non-commercial uses of the work are permitted, provided the original work is properly cited. 
recommended to finish the margins supragingivally. Use of removable prostheses is also troublesome due to the presence of dry and inflamed mucosa [8]. Tooth extraction and subsequent rehabilitation with implants is a good option, especially in young patients who want a fixed prosthesis with long-term prognosis; however, there are some doubts about the use of such prostheses in irradiated patients because of ORN and deficient blood supply. ORN is the irreversible devitalization of irradiated bone. The clinical manifestations of ORN include necrotic soft tissue, orofacial fistulas, pathologic fractures, and suppuration. ORN occurs mostly in the mandible [9]. Trauma-related ORN is usually initiated by tooth extraction [10]. Oral rehabilitation in irradiated patients has always been discussed in the literature, but up to our research, the choice between implant- and tooth-supported prostheses had not been discussed.

Here, we present a treatment sequence and recalls for an irradiated young patient in order to help clinicians make the best decision in similar cases.

\section{CASE REPORT}

An 18-year-old male referred with the chief complaint of poor masticatory function and unsuitable aesthetics. He suffered from Hodgkin's lymphoma (stage II), which was diagnosed 6 years ago and was treated with head and neck radiation therapy (5000 cGY). Intraoral and extraoral examinations were completed (Fig. 1. A and B). Dietary analysis was performed using a 24-hour recall questionnaire; it was found that sugarcontaining food consumption was excessive and frequent. Salivary flow evaluation showed xerostomia; therefore, oral hygiene instructions were delivered, and at-home fluoride and Xylitol products were prescribed for the patient. Dental and radiographic manifestations were as follows:

1. Extensive carious lesions in teeth \#4, \#5, \#6, \#7, \#21, \#23, \#13, \#14, \#15, \#16, \#17, \#18, \#30, and \#31

2. Loss of vertical dimension (VD)

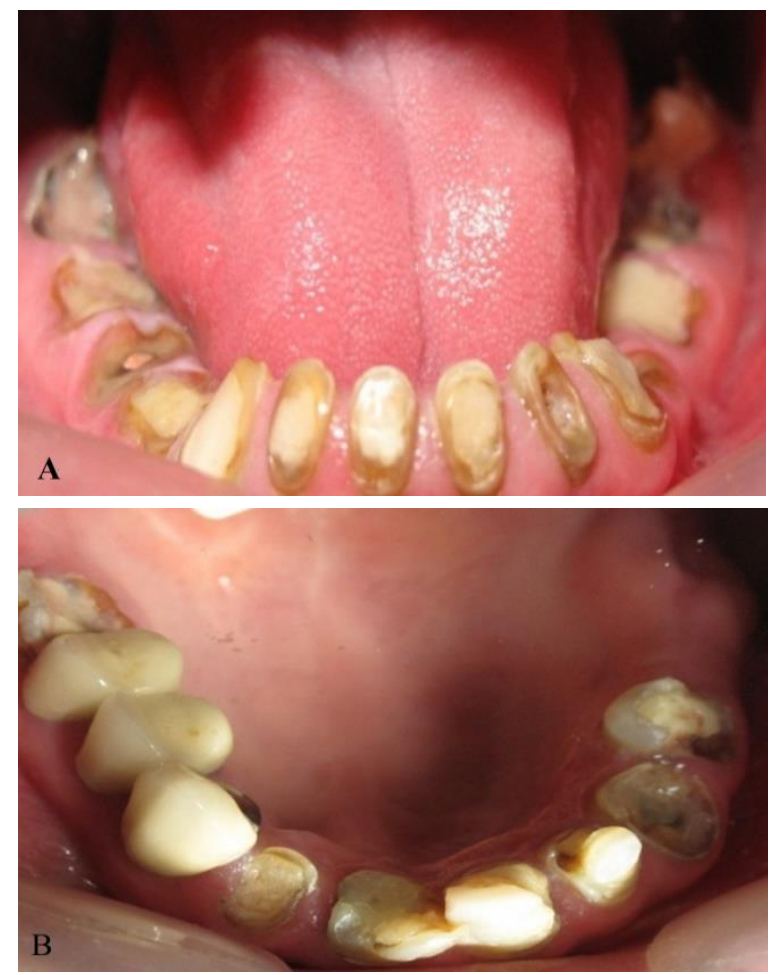

Fig. 1. (A): Intraoral view of the lower jaw. (B): Intraoral view of the upper jaw.

3. Poor oral hygiene

4. Generalized marginal and papillary gingivitis

5. Loss of aesthetics and function

6. Loss of anterior guidance

7. Periapical lesions associated with teeth \#12, \#18, \#30, and \#31

8. Low salivary flow

The diagnostic procedure started with primary cast and record base fabrication for VD analysis. Diagnostic wax-up was done on an articulator for gingival plane and anterior guidance determination (Fig. 2).

The treatment sequences were as follows:

Phase I:

- Oral hygiene instructions and plaque control

- Caries risk assessment

- Root canal treatment of teeth \#4, \#5, \#6, \#7, \#11, \#12, \#21, \#23, and \#28

- Extraction of teeth \#1, \#2, \#13, \#14, $\# 15$, \#16, \#17, \#18, and \#31

- Antimicrobial treatments 


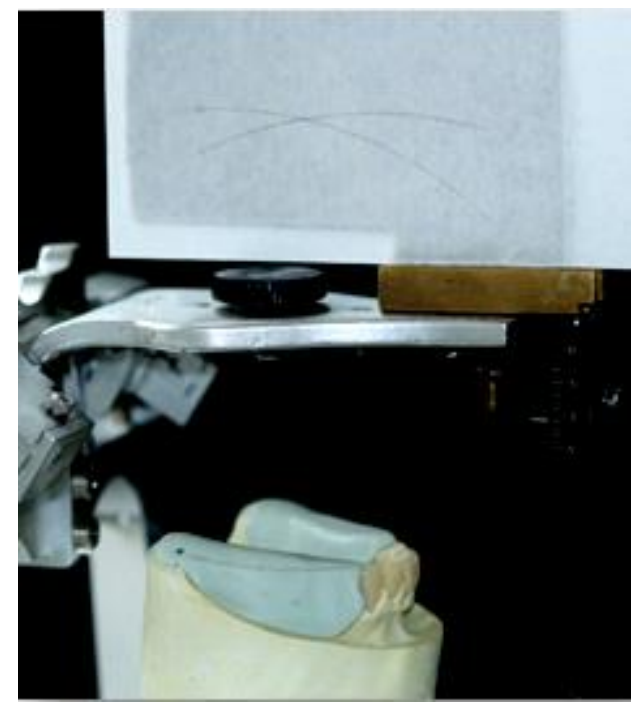

Fig. 2: Determination of the gingival plane and anterior guidance on the diagnostic wax-up

\section{Phase II:}

Crown lengthening

Overlay prosthesis

Phase III:

- Implant placement at the site of teeth \#13 and \#15

- Fixed prostheses (VD reconstruction and full-mouth rehabilitation)

Maintenance (phase IV):

- Periodical recalls:

- Plaque and calculus

- Gingival condition

- Occlusion and mobility

- Other pathologic changes

After gingival plane determination, second wax-up was done on a duplicating cast to fabricate a surgical stent for crown lengthening surgery. An overlay denture was delivered for VD, phonetic, and aesthetic analyses as well as temporary function (Fig. 3)

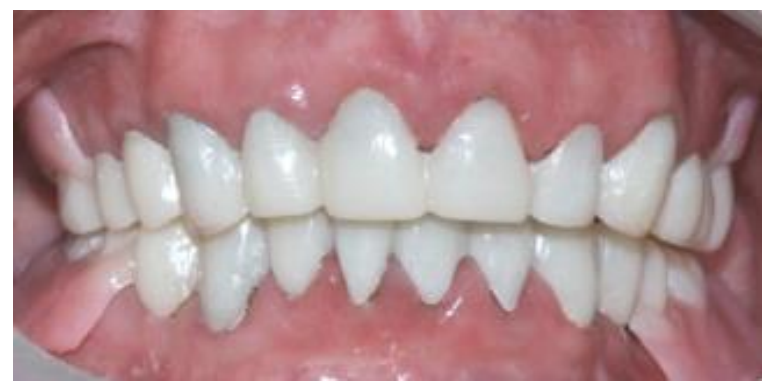

Fig. 3: Overlay prosthesis
Radiographic and surgical stents were fabricated to choose the best site for implant insertion as the diagnostic wax-up indicated (Fig. 4).

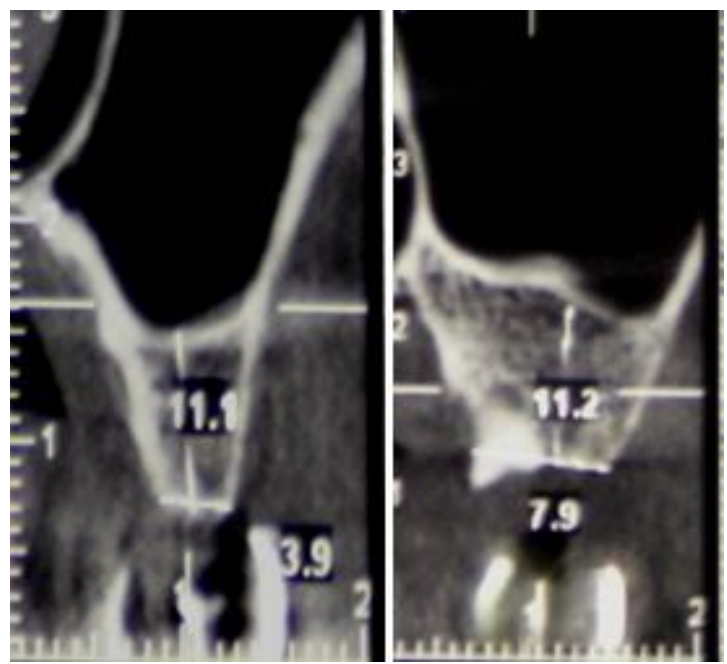

Fig. 4: Cone-beam computed tomographic (CBCT) analysis before implant placement

The implants were installed after making crestal incisions and buccal and lingual flaps. The insertion torque was $50 \mathrm{Ncm}$ (Newtoncentimeters). Delayed installation and delayed loading were chosen. NobelActive ${ }^{\mathrm{TM}}$ implants with grooves (Nobel Biocare Holding AG, Zürich, Switzerland) were used. These implants induce bone formation and improve the initial fixation of the implant, especially in softer bone. By cutting the anterior segment of the overlay denture, a putty index was obtained for post fabrication (Fig. 5).

At first, chairside fixed temporary restorations were made, and after post cementation, the final lab-processed temporary restoration was delivered to the patient (Fig. 6).

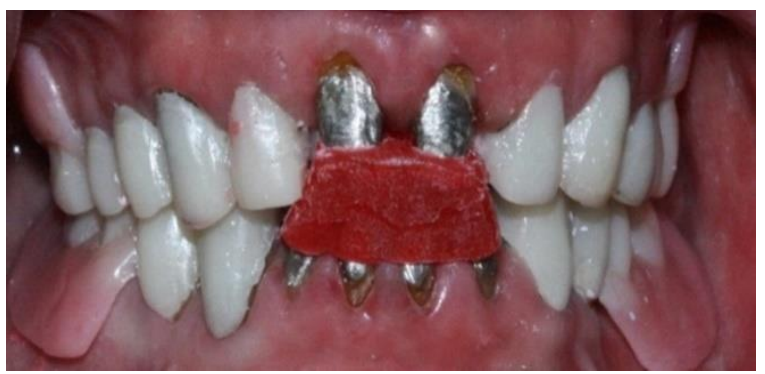

Fig. 5: Anterior segment of the overlay denture used for post fabrication 


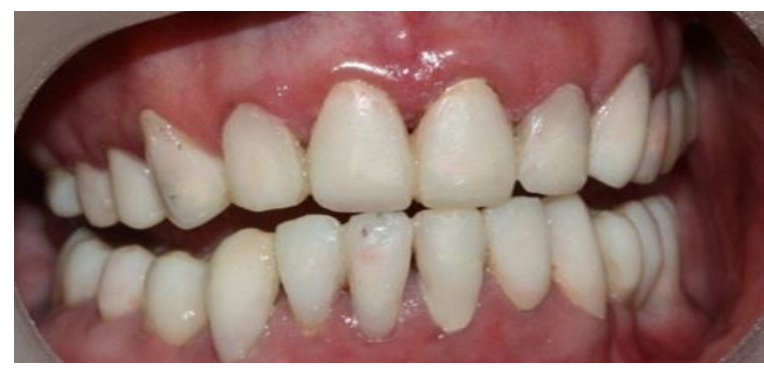

Fig. 6: Temporary restorations in protrusive position

Aesthetics and phonetics were verified. Also, lateral and protrusive movements and amount of disclusion were reviewed. An impression was taken from the prepared teeth, abutments, and temporary restoration, which was transferred to an articulator for cross-mount procedures. Final wax-up and cutback were performed. The putty index was used for the application of porcelain. Framework and porcelain adjustments were performed. The restorations were glazed and cemented. The VD and lateral movements were checked (Fig. 7).

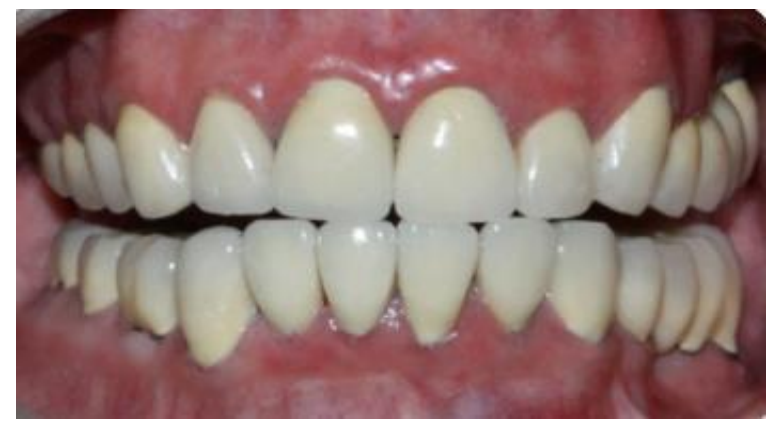

Fig. 7: Final restorations in protrusive position

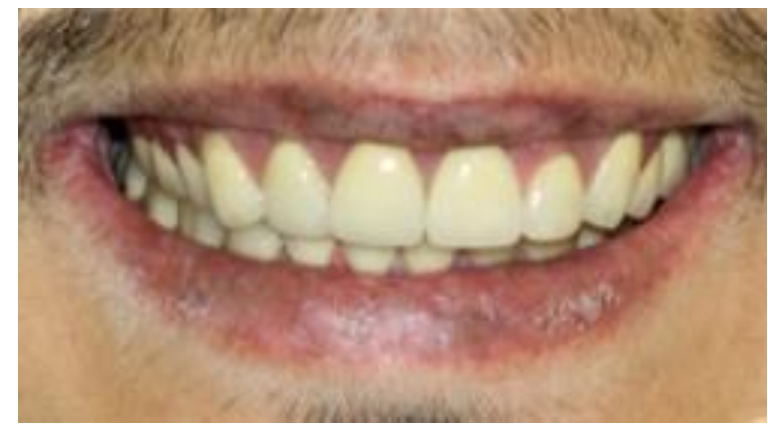

Fig. 8. After 9 months of follow-up

Oral hygiene instructions were emphasized, which included dental flossing after each meal, tooth brushing three times a day, daily use of $0.2 \%$ sodium fluoride (NaF) mouthwash, chewing sugar-free gum, use of saliva substitutes, and periodical periodontic, prosthodontic, and endodontic recalls [10]. The patient attended the 3-month, 6-month, and 9-month recall exams (Fig. 8). The presence of recurrent caries, need for occlusal adjustment, and oral hygiene status were examined. The patient did not attend the 5year recall session. After five years, he referred because some crowns had been detached. Intraoral examinations revealed the loss of most crowns due to the severity of the decay, especially in the mandible. Gingival inflammation and complete destruction of some teeth were detected. The crowns on the implants were intact, and there was no mobility or tenderness in association with the implants. The patient did not return for retreatment; therefore, we could not take intraoral pictures because of ethical considerations.

\section{DISCUSSION}

When discussing prosthetic treatment options, the socioeconomic status of patients and their motivation for treatment should be carefully evaluated. Hygienic habits and lifestyle are important factors in selecting a treatment plan. Even the best treatment will fail with poor maintenance, especially in irradiated patients. In some of these patients, due to numerous medical treatments and psychological problems caused by cancer, there is less willingness to maintain health and attend follow-up sessions. If a patient has low motivation for oral hygiene, more extensive tooth extraction should be considered [11]. Therefore, it is better to choose a treatment that requires less care and attention from the patient. On the other hand, due to the risk of radiation caries, there is a possibility of failure of costly and time-consuming prosthetic treatments even with proper care; this may cause irreparable psychological harm to the patient, thus preventing further compensatory treatment.

Consultation with a dentist is necessary before radiation therapy and must be continued during and after radiotherapy [10]. Evidence suggests that $94 \%$ of the patients consult with a dentist before radiotherapy but only $53 \%$ of 
the patients are under the supervision of a dentist after radiotherapy [12,13]. Requirements for dental extractions before radiation therapy are not universally accepted and depend on each patient's oral condition. However, non-restorable teeth should be considered for extraction before radiotherapy. The periapical, periodontal, and restorative qualification should be carefully examined [14]. Teeth with questionable prognosis should be extracted up to 10-14 days before radiotherapy. However, the situation is complicated for a patient that refers to a dentist after radiotherapy, especially for teeth with questionable prognosis. In these patients, the teeth are prone to recurrent radiation caries, and the gingiva is inflamed due to mucositis. On the other hand, teeth extraction increase the risk of necrosis [15].

Falcao et al [16] demonstrated that a fixed prosthesis is better than removable ones in irradiated patients. Ray-Chaudhuri et al [1] did not recommend the use of conventional bridges in irradiated patients and classified cost/benefit factors for decision-making regarding tooth extraction. Posterior carious teeth in the mandible, xerostomia, trismus, low motivation, and poor hygiene maintenance lead to dental extraction [1].

Use of dental implants in irradiated patients has been discussed in the literature. A reduced implant retention rate has been reported [1719]. Some researchers advised the use of hyperbaric oxygen treatment (HBOT) and reported implant failure only in irradiated bone with free flaps [20]. Some authors stated that HBOT has no benefit for irradiated patients [21].

Falcao et al [16] presented some criteria for successful osseointegration. Atraumatic surgical procedures and stress-reducing prosthodontic treatments are important factors in this respect. [16]. Shaw et al [22] reported that despite some problems with soft tissue, most patients had successful treatment, and radiotherapy failed to affect the treatment prognosis [22].

\section{CONCLUSION}

When facing a patient with radiation caries in need of a fixed prosthesis, if there is no possibility for supragingival placement of the margins of crowns and bridges and when the teeth require costly and time-consuming treatments, such as root canal therapy and post and crown placement, it seems better to consider the replacement of teeth with dental implants.

\section{ACKNOWLEDGMENTS}

The authors would like to thank Dr. Hamid Jalali for supervising the treatment.

\section{CONFLICT OF INTEREST STATEMENT}

None declared.

\section{REFERENCES}

1. Ray-Chaudhuri A, Shah K, Porter RJ. The oral management of patients who have received radiotherapy to the head and neck region. Br Dent J. 2013 Apr;214(8):387-93.

2. Gupta N, Pal M, Rawat S, Grewal MS, Garg H, Chauhan D, et al. Radiation-induced dental caries, prevention and treatment - A systematic review. Natl J Maxillofac Surg. 2015 Jul-Dec;6(2):160-6.

3. Specht L. Oral complications in the head and neck radiation patient. Introduction and scope of the problem. Support Care Cancer. 2002 Jan;10(1):36-9.

4. Naidu MU, Ramana GV, Rani PU, Mohan IK, Suman A, Roy P. Chemotherapyinduced and/or radiation therapy-induced oral mucositis--complicating the treatment of cancer. Neoplasia. 2004 SepOct;6(5):423-31.

5. Aguiar GP, Jham BC, Magalhães CS, Sensi LG, Freire AR. A review of the biological and clinical aspects of radiation caries. J Contemp Dent Pract. 2009 Jul 1;10(4):83-9.

6. Yesilyurt C, Bulucu B, Sezen O, Bulut G, Celik D. Bond strengths of two conventional glass-ionomer cements to irradiated and nonirradiated dentin. Dent Mater J. 2008 Sep;27(5):695-701.

7. Walker MP, Wichman B, Cheng AL, Coster J, Williams KB. Impact of Radiotherapy Dose on Dentition Breakdown in Head and Neck Cancer Patients. Pract Radiat Oncol. 2011;1(3):142-48. 
8. Beech N, Robinson S, Porceddu S, Batstone M. Dental management of patients irradiated for head and neck cancer. Aust Dent J. 2014 Mar;59(1):20-8.

9. David LA, Sàndor GK, Evans AW, Brown DH. Hyperbaric oxygen therapy and mandibular osteoradionecrosis: a retrospective study and analysis of treatment outcomes. J Can Dent Assoc. 2001 JulAug;67(7):384.

10. Hancock PJ, Epstein JB, Sadler GR. Oral and dental management related to radiation therapy for head and neck cancer. J Can Dent Assoc. 2003 Oct;69(9):585-90.

11. Vissink A, Burlage FR, Spijkervet FK, Jansma J, Coppes RP. Prevention and treatment of the consequences of head and neck radiotherapy. Crit Rev Oral Biol Med. 2003;14(3):213-25.

12. Omer O, MacCarthy D, Nunn J, Cotter E. Oral health needs of the head and neck radiotherapy patient: 2 . Oral and dental care before, during and after radiotherapy. Dent Update. 2005 Dec;32(10):575-82.

13. Pradier O, Hille A, Schmiberger $\mathrm{H}$, Hess CF. Monitoring of therapy in head and neck patients during the radiotherapy by measurement of Cyfra 21-1. Cancer Radiother. 2002 Feb;6(1):15-21.

14. Jawad H, Hodson NA, Nixon PJ. A review of dental treatment of head and neck cancer patients, before, during and after radiotherapy: part 1. Br Dent J. 2015 Jan;218(2):65-8.

15. Schiødt M, Hermund NU. Management of oral disease prior to radiation therapy.
Support Care Cancer. 2002 Jan;10(1):40-3. 16. Falcao B, Januzzi E, Santos F. Oral Rehabilitation Following Head and Neck Cancer Treatment-Review of literature. J Palliat Care Med. 2015 Feb;5:208.

17. Ihde S, Kopp S, Gundlach K, Konstantinović VS. Effects of radiation therapy on craniofacial and dental implants: a review of the literature. Oral Surg Oral Med Oral Pathol Oral Radiol Endod. 2009 Jan;107(1):56-65.

18. Granström G. Placement of dental implants in irradiated bone: the case for using hyperbaric oxygen. J Oral Maxillofac Surg. 2006 May;64(5):812-8.

19. Esposito M, Grusovin MG, Patel S, Worthington HV, Coulthard P. Interventions for replacing missing teeth: hyperbaric oxygen therapy for irradiated patients who require dental implants. Cochrane Database Syst Rev. 2008 Jan 23;(1):CD003603.

20. Barrowman RA, Wilson PR, Wiesenfeld D. Oral rehabilitation with dental implants after cancer treatment. Aust Dent J. 2011 Jun;56(2):160-5.

21. Schoen PJ, Raghoebar GM, Bouma J, Reintsema H, Vissink A, Sterk W, et al. Rehabilitation of oral function in head and neck cancer patients after radiotherapy with implant-retained dentures: effects of hyperbaric oxygen therapy. Oral Oncol. 2007 Apr;43(4):379-88.

22. Shaw RJ, Sutton AF, Cawood JI, Howell RA, Lowe D, Brown JS, et al. Oral rehabilitation after treatment for head and neck malignancy. Head Neck. 2005 Jun;27(6):459-70. 